\title{
ON COMPACT DIVISIBLE ABELIAN SEMIGROUPS
}

\author{
JOHN A. HILDEBRANT
}

The algebraic structure of divisible abelian semigroups has been studied in [9] and [1]. Some results on the topological and algebraic structure of compact uniquely divisible abelian semigroups have been obtained in [4] and [5].

A statement of equivalent conditions for a compact abelian semigroup to be divisible is presented in the present paper. Some of the results in [4] are extended to subunithetic semigroups, which are the fundamental building blocks of a divisible abelian semigroup.

\section{Preliminary results.}

Notation. Throughout this paper $N$ denotes the set of all positive integers, $R$ denotes the set of all positive rational numbers, and $R^{-}$ denotes the additive semigroup of all nonnegative real numbers.

Definition. An element $x$ in a semigroup $S$ is said to be [uniquely] divisible if for each $n \in N$ there exists an [unique] element $y \in S$ such that $y^{n}=x$. If each element of $S$ is [uniquely] divisible, then $S$ is said to be [uniquely] divisible.

If $S$ is a semigroup, then $E(S)$ denotes the set of all idempotent elements of $S$.

A semigroup on $[0,1]$ under usual multiplication is called a $U$ semigroup. A semigroup on $[0,1]$ under multiplication defined by $x y=\min (x, y)$ is called an $M$-semigroup. A semigroup on $[(1 / 2), 1]$ with multiplication defined by $x \circ y=\max ((1 / 2), x y)$, where $x y$ is the usual product, is called a $C$-semigroup.

Let $U=[0,1]$ be a $U$-semigroup, $C=[0,1]$ a $C$-semigroup, and $S$ a semigroup. The semigroup $(S \times U) /(S \times\{0\})$ is called the $U$-cone over $S$ and the semigroup $(S \times C) /(S \times\{0\})$ is called the $C$-cone over $S$.

Lemma 1.1. Let $S$ be a compact divisible abelian semigroup. For each $n \in N$, let $S_{n}=S$ and define $T_{n}^{n}: S_{m} \rightarrow S_{n}$ by $T_{n}^{m}(z)=z^{m ! / n !}$ for $m>n$. Then $S^{*}=$ Proj. $\operatorname{Lim}\left(S_{n}, T_{n}^{m}, N\right)$ is a compact uniquely divisible abelian semigroup. Moreover, if $S$ is uniquely divisible, then $S^{*}$ is iseomorphic (topologically isomorphic) to $S$.

Proof. Since $S$ is divisible and abelian, each bonding map $T_{n}^{m}$ is an onto homomorphism. It follows that $S^{*}$ is a compact abelian semigroup.

Received by the editors January 13, 1967. 
Let $x=\left(x_{1}, x_{2}, \cdots\right)$ be an element of $S^{*}$ and $n \in N$. For each $k \in N$, let $m(k)=(n+k-1) ! / k ! n$. Then $m(k) \in N$ for each $k \in N$. Let $y$ $=\left(x_{n}^{m(1)}, x_{n+1}^{m(2)}, \cdots, x_{n+k-1}^{m(k)}, \cdots\right)$. Then $y \in S^{*}$ and $y^{n}=x$. Therefore $S^{*}$ is divisible.

Suppose $a=\left(a_{1}, a_{2}, \cdots\right)$ and $b=\left(b_{1}, b_{2}, \cdots\right)$ are elements of $S^{*}$ and $a^{m}=b^{m}$ for some $m \in N$. Then $a_{j}^{m}=b_{j}^{m}$ for each $j \in N$. Let $i \in N$. Then there exists $p \in N$ such that $m p>i$. Now $a_{m p}^{m}=b_{m p}^{m}$. Hence

$$
a_{i}=a_{m p}^{m p(m p-1) \cdots(i+1)}=b_{m p}^{m p(m p-1) \cdots(i+1)}=b_{i}
$$

and thus $a=b$. It follows that $S^{*}$ is uniquely divisible.

The first projection $\pi$ and indeed every projection of $S^{*}$ to $S$ is onto. If $S$ is uniquely divisible, then $\pi$ is a one-to-one continuous homomorphism of $S^{*}$ onto $S$, and thus, since $S^{*}$ is compact and $S$ is Hausdorff, $\pi$ is an iseomorphism.

Notation. If $S$ is a compact divisible abelian semigroup, then $S^{*}$ denotes the projective limit of the system in Lemma 1.1 and $\pi$ denotes the first projection of $S^{*}$ onto $S$.

Definition. A uniquely divisible semigroup $S$ is said to be unithetic if there exists $x \in S$ such that $S$ is the closure of the set of all positive rational powers of $x$. The element $x$ is called a (unithetic) generator of $S$.

A divisible abelian semigroup $S$ is said to be subunithetic if $S^{*}$ is unithetic. If $x$ is a unithetic generator of $S^{*}$, then $\pi(x)$ is called a (subunithetic) generator of $S$.

THEOREM 1.2. Let $S$ be a compact [uniquely] divisible abelian semigroup and $x \in S$. Then there exists $Q \subset S$ such that $Q$ is minimal with respect to being a compact divisible subsemigroup of $S$ containing $x$. Moreover, $Q$ is [unithetic] subunithetic and generated by $x$.

\section{Subunithetic semigroups.}

THEOREM 2.1. A compact semigroup $S$ is subunithetic if and only if $S$ is the continuous homomorphic image of a compact unithetic semigroup.

Proof. Suppose $S$ is subunithetic. Then $S^{*}$ is unithetic and $\pi: S^{*} \rightarrow S$ is a continuous on to homomorphism.

Suppose $Q$ is a compact unithetic semigroup and $f: Q \rightarrow S$ is a continuous on to homomorphism. Then, by Lemma $1.1, Q^{*}$ is isomorphic to $Q$. Define $f^{*}: Q^{*} \rightarrow S^{*}$ by $f^{*}\left(\left(x_{1}, x_{2}, \cdots\right)\right)=\left(f\left(x_{1}\right), f\left(x_{2}\right), \cdots\right)$. Then $f^{*}$ is a continuous homomorphism of $Q^{*}$ onto $S^{*}$. Let $x$ be a unithetic generator of $Q^{*}$. Then $S^{*}$ is uniquely divisible and $S^{*}$ $=\left[f^{*}(x)\right]$. Hence $S^{*}$ is unithetic and thus $S$ is subunithetic. 
It has been brought to the attention of the author by the referee that the class of compact subunithetic semigroups consists of those compact (submonogenic) semigroups which contain dense algebraic homomorphic images of the nonnegative additive rational numbers (see [6]).

TheOREM 2.2. Let $S$ be a compact subunithetic semigroup which is not a group. Then $S$ is an abelian clan containing exactly two idempotents. The kernel (minimal ideal) $K$ of $S$ and the maximal subgroup $H(1)$ containing the identity (1) of $S$ are subunithetic groups. There is a continuous homomorphism $\psi$ of $R^{-}$into $S$ such that $S=H(1)\left(\psi\left(R^{-}\right)\right)^{-}$ (closure in $S$ ). Moreover, $S / K$ is subunithetic and is either the $U$-cone or the $C$-cone over $H(1)$.

Proof. The first three conclusions follow from [4, Theorem 3.1] and Theorem 2.1 , where $\psi=\pi \sigma$. They can also be established by using the results and methods in [8] or [6]. The last result is a consequence of [4, Theorem 3.4], Theorem 2.1, and the fact that the only subunithetic semigroups on $[0,1]$ are the $U$-semigroup and $C$-semigroup (see [2]).

Definition. A semigroup $S$ is solenoidal if $S$ contains a dense continuous homomorphic image of $R^{-}$(see [7]).

A group $G$ is solenoidal if $G$ contains a dense continuous homomorphic image of the additive real numbers (see [3]).

THEOREM 2.3. Let $S$ be a compact nondegenerate semigroup with identity (1) such that $H(1)=\{1\}$. These are equivalent:

(i) $S$ is subunithetic;

(ii) $S$ is solenoidal;

(iii) $S$ is the continuous homomorphic image of a compact unithetic semigroup.

Proof. The equivalence of (i) and (iii) is established in Theorem 2.1. The fact that (i) implies (ii) is a consequence of Theorem 2.2, where $S=\left(\psi\left(R^{-}\right)\right)^{-}$. The fact that (ii) implies (i) will now be established.

Suppose $S$ is solenoidal and let $\phi: R^{-} \rightarrow S$ be a continuous homomorphism such that $\phi\left(R^{-}\right)$is dense in $S$. Then $S$ is a compact divisible abelian semigroup. Let $\alpha \in R^{-} \backslash\{0\}$ and $x$ $=(\phi(\alpha), \phi(\alpha / 2 !), \phi(\alpha / 3 !), \cdots)$ in $S^{*}$. Let $r=m / n ; m, n \in N$. Then $\pi(r x)=\pi(m x / n)=m \pi(x / n)=m \phi(\alpha / n)=\phi(m \alpha / n)=\phi(r \alpha)$. Thus $\pi(R x)$ $=\phi(R \alpha)$ is dense in $S$. Since $R x$ is dense in $[x]$ (see $[4]), \pi([x])$ is dense in $S$. Therefore, since $\pi([x])$ is closed, $S=\pi([x])$, and thus $S$ is subunithetic. 
Notation. Let $\Sigma$ denote the $a$-adic solenoid with $a=(2,3,4, \cdots)$ (see [3]).

EXAMPLE. The following is an example of a subunithetic semigroup which is not solenoidal: Let $C$ denote the circle group and $U=[0,1]$ a $U$-semigroup. Let $S=C \times U$. Then $S^{*}=\Sigma \times U$, which is unithetic [4, Theorem 4.2]. Hence $S$ is subunithetic but not solenoidal.

THEOREM 2.4. A compact abelian group $G$ is subunithetic if and only if $G$ is solenoidal.

Proof. In [4, Theorem 2.3] it is established that a compact abelian group is unithetic if an only if it is iseomorphic to $\Sigma^{\Delta}$, where card $\Lambda \leqq c$. Therefore, any unithetic group is a continuous homomorphic image of $\Sigma^{c}$. Moreover, by Theorem 2.1, any continuous homomorphic image of $\Sigma^{c}$ is subunithetic. Thus $G$ is subunithetic if and only if $G$ is the continuous homomorphic image of $\Sigma^{c}$. Hence, by [3, Theorem 25.19], $G$ is subunithetic if and only if $G$ is solenoidal.

THEOREM 2.5. Let $S_{1}$ and $S_{2}$ be compact semigroups such that $S_{1} \times S_{2}$ is subunithetic. Then each of $S_{1}$ and $S_{2}$ is subunithetic and either $S_{1}$ or $S_{2}$ is a group.

Proof. Let $Q=S_{1} \times S_{2}$. Then $Q^{*}$ is unithetic. Define $f: Q^{*} \rightarrow S_{1}^{*} \times S_{2}^{*}$ by $\left.f\left(\left(x_{1}, y_{1}\right),\left(x_{2}, y_{2}\right), \cdots\right)\right)=\left(\left(x_{1}, x_{2}, \cdots\right),\left(y_{1}, y_{2}, \cdots\right)\right)$. Then $f$ is an iseomorphism. Thus, by [4, Theorem 4.1], $S_{1}^{*}$ and $S_{2}^{*}$ are unithetic and either $S_{1}^{*}$ or $S_{2}^{*}$ is a group. Hence $S_{1}$ and $S_{2}$ are subunithetic and either $S_{1}$ or $S_{2}$ is a group.

THEOREM 2.6. Let $S$ be a compact first countable subunithetic semigroup and $G$ a compact finite dimensional subunithetic group. Then $G \times S$ is subunithetic.

Proof. Let $Q=G \times S$. Now $S^{*}$ is first countable and unithetic, and $G^{*}$ is finite dimensional and unithetic. Hence, by [4, Theorem 4.2], $G^{*} \times S^{*}$ is unithetic. Since $Q^{*}$ is iseomorphic to $G^{*} \times S^{*}, Q^{*}$ is unithetic. Hence $Q$ is subunithetic.

As in the case for unithetic semigroups, the question as to whether one or both of the conditions that $S$ be first countable and $G$ be finite dimensional can be removed in Theorem 2.6 is open.

3. The semigroup $D(x)$.

Definition. Let $S$ be a semigroup and $x$ a divisible element of $S$. Define $D(x)=\left\{y \in S: y^{m}=x^{n}\right.$ some $\left.m, n \in N\right\}$ - (closure in $S$ ).

THEOREM 3.1. Let $S$ be a compact divisible abelian semigroup and $x \in S$. Then $D(x)$ is a compact divisible semigroup of $S$. 
THEOREM 3.2. Let $S$ be a compact subunithetic semigroup and $x$ a generator of $S$. Then $S=D(x)$.

Proof. Let $y$ be a generator of $S^{*}$ such that $x=\pi(y)$. Let $U$ be an open set in $S$. Then $\pi^{-1}(U)$ is open in $S^{*}=[y]$. Hence there exists $r=m / n, m, n \in N$ such that $y^{r} \in \pi^{-1}(U)$, and thus $\pi\left(y^{m}\right)=(\pi(y))^{m}=x^{m}$. Hence $\pi\left(y^{r}\right) \in U \cap D(x)$. Thus each open set in $S$ meets $D(x)$. Since $D(x)$ is closed, $S=D(x)$.

If $S$ is a compact uniquely divisible abelian semigroup and $x \in S$, then $D(x)$ is the unithetic semigroup generated by $x$. The following is an example of a compact divisible abelian semigroup $S$ containing an element $x$ such that $D(x)$ is not subunithetic:

ExAmple. Let $M$ be an $M$-semigroup with zero, and $U=[0,1]$ a $U$-semigroup. Define the following closed congruence relation on $U \times M: Q=\{((a, b),(c, d)): a=c \leqq 1 / 2$ or $(a, b)=(c, d)\}$. Let $S$ $=(U \times M) / Q$ and $\phi$ the natural map of $U \times M$ onto $S$. Let $x=\phi((3 / 4, z))$. Then $S=D(x)$ and there is exactly one subunithetic subsemigroup $P=\phi(U \times\{z\})$ containing $x$.

EXAMPLE. The following is an example of a compact semigroup $S$ containing a divisible element $x$ such that $S=D(x)$ and $S$ is not divisible: Let $S=[-1,1]$ under usual multiplication and $x=1 / 2$.

\section{Divisible semigroups.}

THEOREM 4.1. Let $S$ be a compact abelian semigroup. Then these are equivalent:

(i) $S$ is divisible;

(ii) each component of $S$ is a divisible subsemigroup;

(iii) each element of $S$ lies on a subunithetic subsemigroup;

(iv) $S$ is the continuous homomorphic image of a compact uniquely divisible abelian semigroup.

Proof. It is immediate that any of conditions (ii), (iii), or (iv) implies condition (i).

(i) implies (ii). Let $C$ be a component of $S$ and $x \in C$. Let $y \in \pi^{-1}(x)$. Then $\pi([y])$ is a subclan of $S$ containing $x$ and hence $\pi([y]) \subset C$. Let 1 denote the identity of $\pi([y])$. Then $x=1 \cdot x \in C x$ and $C x$ is connected. Hence $C x \subset C$ and thus $C$ is a subsemigroup of $S$. Since $x$ has roots in $\pi([y]), x$ has roots in $C$. Therefore $C$ is divisible.

(i) implies (iii). This follows from Theorem 1.2.

(i) implies (iv). This follows from the fact that $S$ is the continuous homomorphic image of $S^{*}$ under $\pi$.

COROLlaRY 4.2. Let $S$ be a compact divisible abelian semigroup such that $S=D(x)$ for some $x \in S$. Then $S$ is connected. 
Proof. Let $T(x)=\left\{y \in S: y^{m}=x^{n}\right.$ some $\left.m, n \in N\right\}$. Let $C$ be the component of $S$ containing $x$. Suppose there exists a component $Q$ of $S$ which is distinct from $C$ such that $T(x) \cap Q \neq \square$. Let $y \in T(x) \cap Q$. Then there exist $m, n \in N$ such that $y^{m}=x^{n}$. Since $C$ and $Q$ are subsemigroups of $S, y \in Q$, and $x \in C, y^{m} \in Q$ and $x^{n} \in C$. Thus $C \cap Q \neq \square$. This contradiction implies that $T(x) \subset C$. Hence $S=D(x)=(T(x))-\subset C$, and thus $S$ is connected.

Definition. An abelian semigroup $S$ such that $S=E(S)$ is called a semilattice.

Corollary 4.3. Let $S$ be a compact totally disconnected divisible abelian semigroup. Then $S$ is a semilattice.

Corollary 4.4. A finite divisible abelian semigroup is a semilattice.

THEOREM 4.5. Let $S$ be a compact abelian semigroup whose subgroups are degenerate. Then $S$ is divisible if and only if each element of $S \backslash E(S)$ lies on a $U$-semigroup or a $C$-semigroup in $S$.

Proof. If each element of $S \backslash E(S)$ lies on a $U$-semigroup or a $C$-semigroup in $S$, then it is immediate that $S$ is divisible.

Suppose $S$ is divisible and $x \in S \backslash E(S)$. Then, by Theorem 1.2, $x$ lies on a subunithetic subsemigroup $Q$ of $S$. It follows from Theorem 2.2 and the fact that the subgroups of $Q$ are degenerate that $Q$ is either a $U$-semigroup or a $C$-semigroup.

\section{REFERENCES}

1. D. R. Brown and J. G. LaTorre, A characterization of commutative uniquely divisible semigroups, Pacific J. Math. 18 (1966), 57-60.

2. H. Cohen and I. S. Krule, Continuous homomorphic images of real clans with zero, Proc. Amer. Math. Soc. 10 (1959), 106-109.

3. E. Hewitt and K. A. Ross, Abstract harmonic analysis, Academic Press, New York, 1963.

4. J. A. Hildebrant, On compact unithetic semigroups, Pacific J. Math. 21 (1967), $265-273$.

5. - On uniquely divisible semigroups on the two-cell, Pacific J. Math. 23 (1967), 91-95.

6. K. H. Hofmann, Topologische Halbgruppen mit dichter submonoger Unterhalb. gruppe, Math. Z. 74 (1960), 232-276.

7. K. H. Hofmann and P. S. Mostert, Elements of compact semigroups, Merrill, Columbus, Ohio, 1966.

8. Anne Lester (Hudson), Some semigroups on the two-cell, Proc. Amer. Math. Soc. 10 (1958), 162-166.

9. T. Tamura, Minimal commutative divisible semigroups, Bull. Amer. Math. Soc. 69 (1963), 713-716.

Louisiana State University 